\title{
Author Correction: Quantification of energy and carbon costs for mining cryptocurrencies
}

Max J. Krause (D) and Thabet Tolaymat

Correction to: Nature Sustainability https://doi.org/10.1038/s41893-018-0152-7, published online 5 November 2018.

In the version of this Analysis originally published, in the paragraph that starts "On the basis of our 2017 estimates...” the word 'trillion' was mistakenly used three times in relation to rates of energy use; it should have read 'billion'. This has now been corrected.

Published online: 16 November 2018

https://doi.org/10.1038/s41893-018-0188-8 